American Journal of Agricultural and Biological Sciences 5 (4): 446-458, 2010

ISSN 1557-4989

(C) 2010 Science Publications

\title{
Genetic and Biochemical Aspects of Ectoine Biosynthesis in Moderately Halophilic and Halotolerant Methylotrophic Bacteria
}

\author{
${ }^{1}$ Valentina N. Khmelenina, ${ }^{1}$ Ildar I. Mustakhimov, ${ }^{1}$ Alexander S. Reshetnikov, \\ ${ }^{2}$ Marina G. Kalyuzhnaya and ${ }^{1}$ Yuri A. Trotsenko \\ ${ }^{1}$ Laboratory of Methylotrophy, \\ G.K. Skryabin Institute of Biochemistry and Physiology of Microorganisms, \\ RAS, Pushchino, Moscow Region, Prospect Nauki 5, Moscow Region, 142290, Russia \\ ${ }^{2}$ Department of Microbiology, University of Washington, Seattle, WA 98115, USA
}

\begin{abstract}
Problem statement: The cyclic imino acid ectoine is a widely distributed compatible solute synthesizing by halophilic and halotolerant bacteria to prevent osmotic stress at high external salinity. This water-keeping compound is used in a variety of commercial cosmetics and therapeutic products. Approach: Development of integrated, predictive functional model of the metabolic and regulatory netwoks of ectoine-producing microbes is an active area of research. In this article we present a brief overview of the current knowledge on genetic and biochemical aspects of ectoine biosynthesis in aerobic halophilic and halotolerant bacteria utilizing $\mathrm{C}_{1}$ compounds (methylotrophs). Although enzymology and genetics of the ectoine biosynthesis in methylotrophs are similar to other halophilic bacteria, the regulatory patterns are different. In all methylotrophic bacteria studied, the genes coding for specific enzymes of ectoine biosynthesis: Diaminobutyric Acid (DABA) aminotransferase (EctB), DABA acetyltransferase (EctA) and ectoine synthase (EctC) are organized into ectABC or ectABC-ask, whith is linked to gene encoding Aspartokinase isozyme (Ask). Results: Remarkably, the methylotrophic bacteria possessing a four-gene cluster showed higher halotolerance and accumulated more ectoine than bacteria with a cluster composed of three genes. The DABA acetyltransferases from three methylotrophic species have been comparatively characterized. The properties of the enzymes correlate with eco-physiological and metabolic particularities of the host. Some elements of the regulatory system governing the ectoine pathway operation have been revealed in both methane and methanol utilizing bacteria. In Methylomicrobium alcaliphilum transcription of the ectABC-ask operon is initiated from two $\sigma^{70}$-like promoters and controlled by the EctR, a MarR-type negative regulator. EctR orthologs were identified in genomes of several heterotrophic halophilic bacteria. Here we present genomic data indicating that similar regulatory system may occur in diverse halophilic and halotolerant bacteria. Conclusion: Currently available data suggest that in methylotrophic bacteria the ectoine biosynthesis pathways are evolutionary well conserved, particularly with respect to the genes and enzymes involved. However, some differences in the ect-gene cluster organization and regulation could be observed.
\end{abstract}

Key words: Halophilic bacteria, osmoadaptation, ectoine, enzymes, transcriptional regulation

\section{INTRODUCTION}

The ability of microbial cells to adapt to fluctuations of external osmolarity (osmoadaptation) via activation of specific mechanisms (osmoregulation) is essential for their survival. Halophilic and halotolerant microorganisms have evolved a number of metabolic, enzymatic and regulatory mechanisms that prevent cell dehydration. Osmoadaptation based on accumulation of inorganic ions, mostly $\mathrm{K}^{+}$(salt type of osmoadaptation), is employed in extremely halophilic members of Halobacteriaceae and Halanaerobiales, as well as anaerobic acetogenic species of Halobacteroides, Sporohalobacter, Acetohalobium and sulfate reducers (Desulfovibrio halophilus, Desulfohalobium retbaense) (Zhilina and Zavarzin, 1990; Galinski and Truper, 1994). Structural and metabolic organization of cells of extremely halophilic

Corresponding Author: Valentina N. Khmelenina, G.K. Skryabin Institute of Biochemistry and Physiology of Microorganisms, RAS, Pushchino, Prospect Nauki 5, Moscow Region, 142290, Russia Tel: +7 4959257448 Fax: +7 4959563370 
bacteria is well adapted to high intracellular concentrations of ions. Moreover, it often depends on high salinity for optimal operation. For example, malate dehydrogenase of Halobacterium salinarum and halophilic archaeon Haloarcula marismortui is more acidic and require $\mathrm{K}^{+}$for compact folding and enzyme activity (Marhuenda-Egea and Bonete, 2002; Mevarech et al., 2000). Another osmoadaptation strategy is based on synthesis of small organic molecules (osmolytes). It occurs in a majority of bacteria, archaea, fungi, plants and even animals (Galinski and Truper, 1994; da Costa et al., 1998; Ventosa and Nieto, 1995). Osmolytes (also known as compatible solutes) do not interfere with cellular metabolism. They are represented by compounds of different classes: (i) zwitterions (amino acids and their derivatives including ectoines and betaines), (ii) neutral solutes (sugars and polyols) and (iii) anionic solutes where the negative charge is supplied by a carboxylate, phosphate or sulfate (Galinski, 1995; Roberts, 2004; 2005). Many bacteria, including non-halophilic, accumulate compatible solutes at hyperosmotic conditions either through de novo synthesis or by uptake from surrounding environment.

Ectoine, a cyclic imino acid (1,4,5,6-tetrahydro-2methyl-4-pyrimidine carboxylic acid) was originally discovered as a compatible solute in an anoxygenic phototrophs of the Ectothiorhodospira group (Galinski et al., 1985). Later, it was found in many aerobic halophilic and halotolerant gram-negative and gram-positive bacteria including Nocardiopsis, Brevibacterium, Marinococcus, Halomonas, Pseudomonas and Vibrio (Ventosa and Nieto, 1995; Galinski, 1995; Kempf and Bremer, 1998; Grant, 2004; Severin et al., 1992). Ectoine and its hydroxylated derivative, hydroxyectoine, are powerful multifunctional bioprotectants that defend against a variety of damaging factors such as heating, freezing, desiccation and UV radiation (Jebbar et al., 1992; Buenger and Driller, 2004; Graf et al., 2008). Both compounds are widely used in medicine and cosmetics as brought-range stabilizers. In spite of a rising commercial interest the genetics and biochemistry of ectoine biosynthesis are intensively studied in various bacteria. We refer here to the genetic and enzymatic aspects of the ectoine biosynthesis pathway in aerobic methylotrophic bacteria which utilize single carbon compounds (methane, methanol or methylamines) as the carbon and energy sources. These moderately halophilic and halotolerant methylotrophs have been isolated from soda lakes and (hyper) saline environments. The members of the genera Methylomicrobium, Methylobacter, Methylophaga
(Gammaproteobacteria) were shown to synthesize ectoine as an osmoprotectant along with sucrose and glutamate (Khmelenina et al., 1999; Doronina et al., 2003a; 2003b; Kalyuzhnaya et al., 2001; 2008). Ectoine was also identified in cells of Methylarcula species (Alphaproteobacteria) grown at high salinity (Doronina et al., 2000).

\section{MATERIALS AND METHODS}

Genetic aspects of ectoine biosynthesis: The ectoine biosynthesis represents a branch of the metabolic road for aspartate family amino acids synthesis (Fig. 1) and involves three special enzymes: Diaminobutyric Acid (DABA) aminotransferase (EctB) that catalyses amination of aspartate semialdehyde into diaminobutyric acid, DABA acetyltransferase (EctA) acetylating DABA into $\mathrm{N} \gamma$-acetyl diaminobutyric acid and ectoine synthase (EctC) that forms ectoine by cycling of $\mathrm{N} \gamma$-acetyl diaminobutyric acid (Peters et al., 1990). In various halophilic bacteria studied, the genes coding for the enzymes form a cluster ectABC (Canovas et al., 1998; Louis and Galinski, 1997; Goller et al., 1998; Kuhlmann and Bremer, 2002). Some bacteria possess an additional enzyme that converts ectoine to hydroxyectoine (ectoine hydroxylase, encoded by ectD) (Canovas et al., 1999; Prabhu et al., 2004; Bursy et al., 2007). The ect-genes are usually, but not always, organized into more or less compact operon.

Analysis of complete microbial genomes showed that the ect-genes are widespread among Proteobacteria and Actinobacteria (Fig. 2). The genetic signature of the ectoine pathway, an ectABC operon, was also identified in genome of Nitrosopumilus maritimus, a nonthermophilic archaeon isolated from a salt-water aquarium. Rather high homology of the ectoine biosynthetic genes in microorganisms of different taxonomic position and physiology is a reliable indication of the evolutionary conservation of the biochemical pathway (Kuhlmann and Bremer, 2002).

High homology of the ect-genes in microorganisms allowed identification of the genes in some aerobic methylotrophs by using PCR-surveys (Reshetnikov et al., 2006). ectABC or ectABC-ask clusters were identified in six species of genera Methylomicrobium, Methylobacter, Methylophaga and Methylarcula. The organization of the ect-genes (three or four gene cluster) correlates with halotolerance of the host strain. Two halotolerant methanotrophs, Methylomicrobium kenyense AMO1 and Methylobacter marinus $7^{\circ} \mathrm{C}$ possess three gene operon (ectABC), accumulate ectoine up to $70 \mathrm{mg} \mathrm{g}^{-1}$ of Dry Cell Weight (DCW) and are capable of growth at salinity $4-5 \% \quad \mathrm{NaCl}$. 


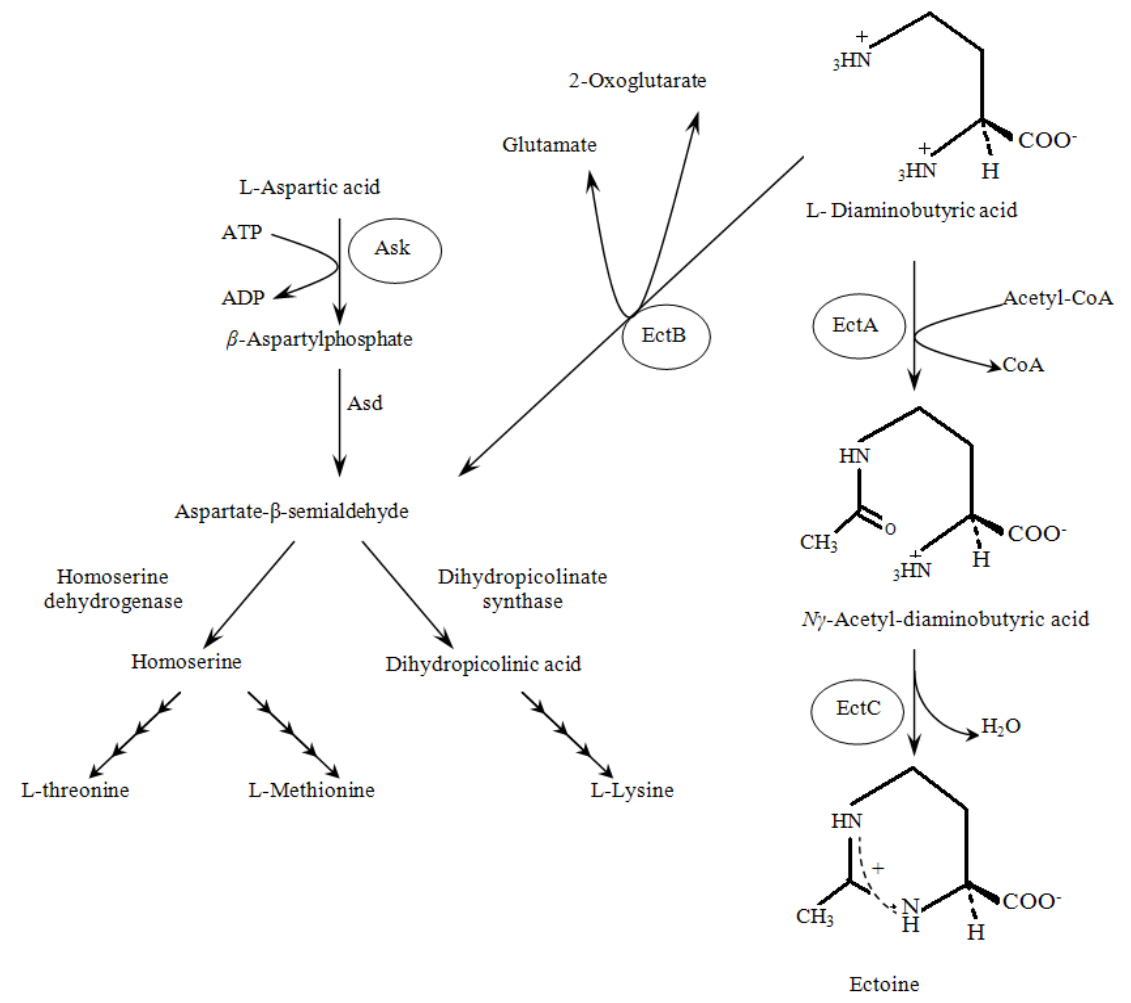

Fig. 1: Pathway of ectoine biosynthesis from aspartic acid

In contrast, methanotrophic species Methylomicrobium alcaliphilum and methanol- and methylamine-utilizers Methylophaga alcalica, Methylophaga thalassica and Methylarcula marina possess ectABC-ask operon, can grow at two-fold higher salinity $(10-12 \% \mathrm{NaCl})$ and accumulate $>20 \mathrm{mg}$ of ectoine $\mathrm{g}^{-1}$ of DCW. This implies an important role of the ectoine pathway specific aspartokinase in some methylotrophic species. It was demonstrated that in Mm. alcaliphilum, genes ectABC and ask are co-transcribed and thus the aspartokinase must be osmotically controlled (Reshetnikov et al., 2006). One could speculate that the occurrence of the aspartokinase isozyme may provide an advantage at high osmolarity as it makes ectoine biosynthesis independent from complex machinery regulating the amino acid biosynthesis. However, in this case a culture must possess an additional enzyme that could fulfill the pool of amino acid for protein synthesis at low salinity. Indeed, a copy of gene coding for aspartokinase with $77 \%$ identity of the translated amino acids is present in $\mathrm{Mm}$. alcaliphilum as followed from a draft genome sequence analyses (https://www.genoscope.cns.fr/agc/mage).

Analyses of the publicly available complete microbial genomes showed that at least 30 bacterial species harbor homologs of the ectABC-ask genes. Bacteria possessing an ask gene immediately downstream of ectABC are exclusively gram-negative. In the majority of the proteobacterial halophiles, a second, additional copy of the aspartokinase gene was identified. Two and three additional ask genes not linked to the ect-gene cluster occur in Photobacterium profundum and Vibrio cholerae, respectively. Interestingly, no aspartokinase gene outside of the ectcluster was found in three species of marine bacteria, Oceanobacter sp. RED65, Sphingomonas sp. SKA58 and Lentisphaera araneosa. In these bacteria the sole ectoine-associated Ask should support both cellular processes-osmoresistance and protein biosynthetic needs. Most likely in these marine bacteria the ectoperon and linked ask gene are constitutively expressed. However, this hypothesis remains to be tested by the comprehensive description of the gene expression and the enzyme properties.

It should be mentioned that in gram-positive bacteria ectoine is not the sole osmoprotectant and other organic compatible solutes belonging to the glutamate family aminoacids, glutamate, glutamine and/or proline contribute to osmotic balance (Kuhlmann and Bremer, 2002; Lo et al., 2009; Saum and Muller, 2007; 2008a; 2008b). 


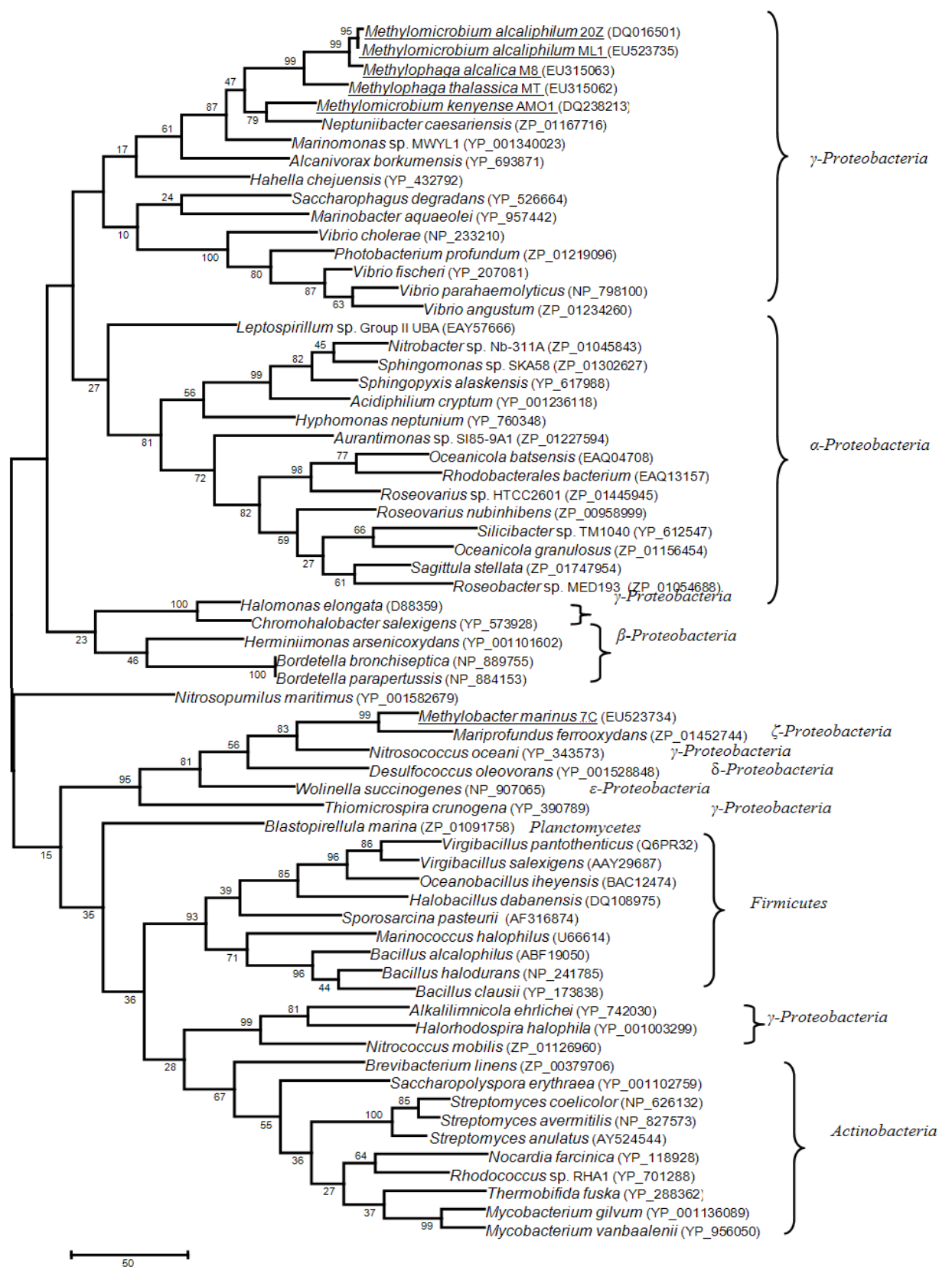

Fig. 2: Phylogenetic tree based on deduced amino acid sequences from ectB genes of methylotrophs(underlined) and those from other bacteria possessing ect genes. The tree was constructed by minimal evolution method. In bracket the accession numbers of the respective genes and whole genomes presented in Database

This may require a complex regulation since the carbon flow must be also directed to biosynthesis of the osmoprotectants not derived from aspartic acid. It may explain the absence of the ask homologues in the ectgene cluster of gram-positive bacteria. Remarkably, the halophilic Firmicutes typically possess multiple ask homologues not linked to the ect-genes (Lo et al., 2009).
Phylogenetic relationships of the EctB from bacteria of different physiological groups are shown in Fig. 2. The topology of the EctB tree was similar to those of EctA- and EctC-based phylogenetic trees (data not shown). In general, the Ect proteins from bacteria belonging to the Alpha- and Gammaproteobacteria, Firmicutes and Actinobacteria comprise separate 
branches on the trees. This may imply an ancient acquisition of the ect-genes from common ancestor and prolonged evolution inside the respective microbial phylum. Interestingly, the Ect proteins of methane- and methanol-utilizing bacteria of the Methylomicrobium and Methylophaga genera are combined in the coherent group on the tree being most closely related to other representatives of the Gammaproteobacteria. On the other hand, the Ect proteins from Mm. kenyense AMO1 are more identical to those of the methanol-utilizers than other methanotrophs. Since the bacterium carries the ect $\mathrm{ABC}$ gene cluster, a recent loss of the ask gene may be proposed. Almost identical Ect proteins (94.8, 98.2 and $98.5 \%$ for EctA, EctB and EctC) are present in two strains of the Mm. alcaliphilum, 20Z and ML1, isolated from the Tuva soda lake (Russia) and Mono Lake (USA), respectively. Hence, geographical distance of the bacterial habitats has not resulted in significant divergence of the ect genes.

Notably, Ect proteins from $M b$. marinus $7^{\circ} \mathrm{C}$, a methanotroph belonging to the Gammaproteobacteria, are only distantly related to ectoine biosynthetic enzymes of other methylotrophs (only 37-51\% identities to EctABC sequences of Mm. alcaliphilum 20Z) and fall with proteins of the marine representatives of Proteobacteria. Ectoine biosynthesis proteins from $\mathrm{Mb}$. marinus $7^{\circ} \mathrm{C}$ are closely related to those from the marine Mariprofundus ferrooxydans (Zetaproteobacteria) (55-80\% identities). However, in $M$. ferrooxydans the ectoine biosynthetic pathway is encoded by an ectABC-ask operon, while the ectcluster from strain 7C lacks ask gene. So far, $M b$. marinus is the only representative of the Methylobacter genus that is salt-resistant and synthesizes ectoine. Other described species of the genus are non-halophilic. It could be proposed that the ect-operon ubiquity in ancient prokaryotic world that was largely marine, followed by loss in lineages that became adapted to a terrestrial environment (Lo et al., 2009). It is also possible that an ancestor, terrestrial species of Methylobacter adapted to marine ecosystem by acquiring ectoine biosynthesis genes from phylogenetically distant species.

The biosynthesis of hydroxyectoine proceeds via direct hydroxylation of ectoine catalyzed by ectoine hydroxylase (EctD) (Bursy et al., 2007; Reuter et al., 2010). In Mm. alcaliphilum, an orf with a high homology to ectD (40-42\% identity to ectoine hydroxylases from Streptomyces avermitilis MA-4680 and $S$. chrysomallus) was identified 360 bp downstream of the ectABC-ask operon (Reshetnikov et al., 2006). No hydroxyectoine accumulation was detected in $\mathrm{Mm}$. alcaliphilum cells grown in the presence of 3 or $9 \%$
$\mathrm{NaCl}$. However, it does not exclude that the methanotroph can produce the bioprotectant at some environmental perturbations. NR-Database searches revealed that genomes of 67 proteobacteria and one archaeon, Nitrosopumilus maritimus, contain homologues of the ectD gene (with identities ranging from $41-55 \%$ to that of $V$. salexigens) (Reuter et al., 2010). ectD-like gene is either a part of the ect-cluster or it locates separately. Majority of the microbial genomes contain only one copy of the ectD gene, with exception to Rhodococcus opacus B4, Marinobacter aquaeolei VT8 and C. salexigens, that possess two types of EctD-proteins (Reuter et al., 2010). In the case of $C$. salexigens, only one of the EctD-like enzymes contributes to the production of 5-hydroxyectoine (Garcia-Estepa et al., 2006) and the ectD gene is essential for thermoprotection of the bacterium.

It should be mentioned that an alternative pathway for transformation of $\mathrm{N} \gamma$-acetyl DABA into hydroxyectoine via 3-hydroxy-N $\gamma$-acetyl DABA without ectoine formation step was proposed but this was not enzymatically proved (Canovas et al., 1999).

\section{RESULTS AND DIACUSSION}

Some properties of the ectoine biosynthetic enzymes: In the conditions of hyperosmotic stress (from 0.5-1 M $\mathrm{NaCl}), \quad H$. elongata cells with blocked by chloramphenicol protein biosynthesis, accumulated ectoine at the same level as the cells with active protein synthesis. Thus, the regulation of ectoine synthesis can proceed at the enzyme level (Kraegeloh and Kunte, 2002). Three ectoine biosynthesis specific enzymes have been purified from $H$. elongata DSM2581 (Ono et al., 1999). The enzymes are characterized by similar parameters for the maximal activity $(\mathrm{pH} 8.2-9.0$, $\mathrm{t}=15-20^{\circ} \mathrm{C}$ and $0.4-0.5 \mathrm{M} \mathrm{NaCl}$ ). Since the optimal salt concentrations for the enzymes were lower than those in the medium supporting maximal growth rate of the bacteria, relatively low intracellular concentrations of ions should be maintained (Kraegeloh and Kunte, 2002). Indeed, $\mathrm{Na}^{+}$content of 0.04-0.2 $\mathrm{M}$ was found in cells of Vibrio costicola и Brevibacterium sp. growing at high $\mathrm{NaCl}$ concentrations (Gilboa et al., 1991; Nagata et al., 1995).

DABA aminotransferase (EctB, EC 2.6.1.76): DABA aminotransferase from $H$. elongata is a homohexameric $(\sim 250 \mathrm{kDa})$ pyridoxal phosphate-dependent enzyme that requires $\mathrm{K}^{+}$for activity and stability. The enzyme is more active in the presence of $0.01-0.5 \mathrm{M} \mathrm{KCl}$, than in the presence of $\mathrm{NaCl}$ (Ono et al., 1999). $\mathrm{K}^{+}$requirement is inherent to many enzymes from extremely halophilic 
eubacteria and archae with the salt-type osmoadaptation (Marhuenda-Egea and Bonete, 2002; Mevarech et al., 2000; Toney et al., 1995). DABA aminotransferase is specific to L-glutamate, as an amino donor $\left(\mathrm{K}_{\mathrm{m}} 9.1\right.$ $\mathrm{mM}$ ) and to $\mathrm{D}$, L-aspartyl semialdehyde $\left(\mathrm{K}_{\mathrm{m}} 4.5 \mathrm{mM}\right)$. The reaction catalyzed by DABA aminotransferase was predicted to be a limiting step of the ectoine biosynthesis pathway thus explaining the absence of DABA in cells of $H$. elongata KS3 (Ono et al., 1999). Two putative DABA aminotransferase genes, one in ectABC-ask operon and another in a cluster of genes presumably involved in ectoine degradation, were found in the genome of $M$. alcaliphilum.

Interestingly, DABA aminotransferase was detected in several bacteria that do not synthesize ectoine. In Acinetobacter baumannii, DABA aminotransferase is involved in biosynthesis of diamonopropane, a component of cell wall peptidoglycan (Ikai and Yamamoto, 1997). In Paenibacillus polymyxa the enzyme is a part of the polymyxin, a peptide antibiotic, formation (Ono et al., 1999). In A. baumannii, it is specific to L-glutamate, whereas in Xanthomonas species to L-alanine as the amino donor (Rao et al., 1969).

DABA acetyltransferase (EctA, EC 2.3.1.178): DABA acetyltransferase from $H$. elongata was purified $(\approx 400$ fold) and was only partially characterized due to low stability of the enzyme (Ono et al., 1999). Histagged DABA acetyltransferases were purified from Mm. alcaliphilum 20Z, M. alcalica and M. thalassica (Reshetnikov et al., 2005; Mustakhimov et al., 2008). The enzyme from these methylotrophs existed as a homodimer with the subunit molecular mass of $\sim 20$ $\mathrm{kDa}$ and had no requirement for divalent ions.

Some differences in the enzyme properties that correlated with eco-physiologies of these bacteria were described. Thus, the DABA acetyltransferase from the neutrophilic $H$. elongata and $M$. thalassica was more active at lower $\mathrm{pH}(\mathrm{pH} 8.2$ or $\mathrm{pH} 9.0)$, than that from alkaliphilic species $\mathrm{Mm}$. alcaliphilum and $M$. alcalica (pH optima $\geq 9.5$ ). Unlike enzymes from $M$. alcalica and $M m$. alcaliphilum, DABA acetyltransferase from the marine bacterium $M$. thalassica was considerably inhibited by carbonates (Mustakhimov et al., 2008). Bearing in mind that growth of the former alkaliphilic methylotrophs is stimulated by carbonates, this feature of the enzyme corresponds to in situ surroundings of the strains. Interestingly, $1 \mathrm{mM} \mathrm{Cu}{ }^{2+}$ completely inhibited activity of the DABA acetyltransferase from $M$. alcalica and $47 \%$ that of $M$. thalassica. In contrast, no inhibitory effect of $\mathrm{Cu}^{2+}$ was found for the enzyme from methanotroph $\mathrm{Mm}$. alcaliphilum and this also correlates with an important role of copper in methane oxidation in the culture, since $\mathrm{Cu}$ is an essential component of the particulate methane monooxygenase (Murrell et al., 2000).

Another intriguing feature of DABA acetyltransferases from methylotrophic bacteria was the effect of ionic strength on enzyme activity. Like the enzyme from $H$. elongata, DABA acetyltransferase from methanotroph $\mathrm{Mm}$. alcaliphilum was activated by $0.2 \mathrm{M} \mathrm{NaCl}$ implying the "halophilic nature" of the protein. Contrary, the enzymes from methylotrophic cultures, $M$. thalassica and $M$. alcalica were inhibited by salts. We may speculate that methanol is more effective substrate for supporting ion extrusion mechanisms in comparison to methane or glucose. As a result, low concentrations of monovalent inorganic ions could be maintained in cytoplasm of the methanolutilizing bacteria eliminating need for ectoine biosynthetic enzymes that are adapted to high ionic strength.

Ectoine synthase (EctC, EC 4.2.1.108): The homogenous ectoine synthase from $H$. elongata was purified in the presence of $1 \mathrm{mM} \mathrm{N} \gamma$-acetyl-DABA and $2 \mathrm{M} \mathrm{NaCl}$ as stabilizing compounds. The molecular mass of the native enzyme remains unclear due to loss of the enzyme activity after gel-filtration at $0.5 \mathrm{M}$ $\mathrm{NaCl}$. The EctC-protein contains enhanced levels of aspartate and glutamate. The enzyme is specific to $\mathrm{N} \gamma$ acetyl-DABA, however the $\mathrm{N}$-acetyl group in $\alpha$ position could not be involved in the cycling process (Ono et al., 1999). The recombinant ectoine synthase was purified from $\mathrm{Mm}$. alcaliphilum $20 \mathrm{Z}$ with activity $\sim 64 \mathrm{U} \mathrm{mg}^{-1}$. The protein is a $35 \mathrm{kDa}$ homodimer.

Ectoine hydroxylase (EctD, EC 1.14.11): Was purified from the moderate halophile Virgibacillus (Salibacillus) salexigens (Bursy et al., 2007) and from the Streptomyces coelicolor (Bursy et al., 2008). EctD from $V$. salexigens is a monomeric protein of molecular mass $34 \mathrm{kDa}$ being a member of the non-heme iron (II)and 2-oxoglutarate-dependent dioxygenases. The reaction depends on iron (II), molecular oxygen and 2oxoglutarate. The similar properties were observed for enzyme from Streptomyces coelicolor with respect to optimal $\mathrm{pH}(\mathrm{pH} 7.5)$, temperature $\left(32^{\circ} \mathrm{C}\right), \mathrm{K}_{\mathrm{m}}$ values for ectoine (3.5 and $2.6 \mathrm{mM})$ and co-substrate 2oxoglutarate (5.2 and $6.2 \mathrm{mM})$. Contrary to preferential production of 5-hydroxyectoine by $S$. coelicolor grown at $39^{\circ} \mathrm{C}$ (Bursy et al., 2007), the high temperature optimum of the EctD enzyme implies that there is no specific thermoactivated regulation of the hydroxylase. 
The crystal structure analysis of the $V$. salexigens EctD (Reuter et al., 2010) showed that the folding of the protein is similar to the human phytanoyl-CoA 2hydroxylase (McDonough et al., 2005). The core of the EctD structure consists of a double-stranded $\beta$-helix forming the main portion of the active site of the enzyme. The positioning of the iron ligand in the active site of EctD is mediated by an evolutionarily conserved 2-His-1-carboxylate iron-binding motif. The side chains of the three residues forming this iron-binding site protrude into a deep cavity in the EctD structure that also harbors the 2-oxoglutarate binding site. Despite high homology and similar reactions catalyzed by the ectoine hydroxylase and L-proline hydroxylase (Hausinger, 2004), the EctD from S. coelicolor had no additional L-proline hydroxylase activity (Bursy et al., 2007).

Transcriptional regulation of the ectoine biosynthesis genes:

Regulation of the ectoine biosynthesis genes in heterotrophic bacteria: To date, there are only fragmentary data on how an external salinity regulates expression of ectoine biosynthetic genes. In Bacillus pasteurii (Kuhlmann and Bremer, 2002), Marinococcus halophilus (Bestvater and Galinski, 2002), Halobacillus halophilus (Saum and Muller, 2008b) and S. salexigens (Bursy et al., 2007), transcription of the ectABC-ask or ect $A B C$ genes proceeded as polycistronic mRNAs and subjected to osmotic conditions. The osmoregulated expression of ectABC was also revealed in gramnegative Brevibacterium epidermis (Onraedt et al., 2004), Mm alcaliphilum (Reshetnikov et al., 2006; Mustakhimov et al., 2010) and Chromohalobacter salexigens (Calderon et al., 2004).

In $C$. salexigens, the ect $A B C$ operon is transcribed from several promoter regions. Four putative promoters (PectA1-4) are located upstream of the ectA gene and one internal promoter PectB is upstream of the ectB gene (Calderon et al., 2004). The consensus sequences of the ect promoters closely resembled those of $\sigma^{70}$ (PectA1 and PectA2), $\sigma^{\mathrm{S}}-($ PectA 3$)$ and $\sigma^{32}-($ PectB) dependent promoters of E. coli. Both PectA and PectB promoter regions were shown to be osmoregulated. Transcription from the PectB was also enhanced upon increasing growth temperature. Expression of the reporter gene lacZ under control of the PectA in recombinant $C$. salexigens and $E$. coli cells was considerably increased when the cultures approached stationary growth phase. Moreover, in the mutant $E$. coli lacking the rpoS gene, the expression of the PectAlacZ fusion was much lower than in the wild type strain. This was consistent with maximal ectoine accumulation in the stationary growth phase (Canovas et al., 1999). It could be reasonable to propose an involvement of the general stress factor $\sigma^{\mathrm{S}}$, however, no rpoS-like gene has been identified in $C$. salexigens (Calderon et al., 2004). Participation of different transcriptional sigma factors in expression of the ect-genes was not confirmed by an appropriate genetic study. Supplementation of the growth media with ectoine or glycine betaine decreased the transcription of PectA-lacZ and PectB-lacZ (Calderon et al., 2004). Altogether with constitutive ect-genes transcription from PectA and PectB at low osmolarity, this implies an involvement of other transcriptional factors.

The recent studies have shown a connection between the iron homeostasis and the osmoresponse in C. salexigens (Argandona et al., 2010). An iron homeostasis regulator, Fur, has been described as a potential regulatory link between salinity and iron metabolism. Corresponding six-gene operon cfuABCfur-hisI-orf6 was identified downstream of the ectABC genes. Fur boxes were found in promoters of the cfuABC-fur-hisI-orf6 and ectABC operons. It was shown that Fur mediates the osmoregulated inhibition by iron of cfuABC-fur-hisI-orf6 expression and functioned as a positive regulator of the ectABC genes under high-salinity conditions.

In gram-positive bacteria, ectoine biosynthesis also seems to be triggered by an osmotic stress. The vegetative $\sigma^{\mathrm{A}}$-dependent promoter (equivalent to $\sigma^{70}$ like promoter of gram-negative bacteria) was found upstream of the ectABC in Bacillus subtilis, $B$. pasteurii and $S$. salexigens (Kuhlmann and Bremer, 2002; Bursy et al., 2007). In Marinococcus halophilus, the transcription of ectABC genes was initiated from three individual $\sigma^{70} / \sigma^{\mathrm{A}}$-promoters located upstream of each gene (Bestvater and Galinski, 2002). The $\sigma^{70} / \sigma^{\mathrm{A}}$ promoter sequences were amplified and cloned upstream of gfp in vector pBR322. A linear increase in fluorescence of $E$. coli cells upon increase of $\mathrm{NaCl}$ concentration in the medium was detected, however, the addition of ectoine and betaine decreased the fluorescence signal (Bestvater and Galinski, 2002).

Moderately halophilic Halobacillus halophilus served as a prominent model bacterium to decipher regulatory mechanisms of the osmolytes biosynthesis. When grown at moderate salinity $(1 \mathrm{M} \mathrm{NaCl}), H$. halophilus accumulated glutamate and glutamine as the major compatible solutes whereas ectoine and proline were predominantly produced at very high salinities (Saum and Muller, 2008a; 2008b). Ectoine/proline ratio that was low in cells growing exponentially at different salinities ( 2 and $3 \mathrm{M} \mathrm{NaCl}$ ) enhanced more than 1000fold in the stationary growth phase. The proline 
exhausting in the stationary phase cells (the amino acid proline can be also used as the carbon or energy source) may be the major reason for the observed growth phasedependent switches (Saum and Muller, 2008a; 2008b).

Osmotic up-shock of $H$. halophilus cells led to the enhanced levels of mRNAs of ectABC and gdh1, glnA2 and proH genes encoding respectively glutamate dehydrogenase, glutamine synthetase and pyrroline-5carboxylate reductase.. The expression of ect-genes reached the maximal level when concentration of transcripts for genes for glutamate, glutamine and proline biosynthesis returned almost to the initial levels. The ectABC transcript concentration was highest in the presence of $\mathrm{NaNO}_{3}$. Na-gluconate was as efficient as $\mathrm{NaCl}$ while Na-glutamate led only to a minor increase of the transcripts. Hence, transcription of the ect-genes did not necessarily depend on the presence of chloride anions (Saum and Muller, 2008b).

It was demonstrated that in $C$. salexigens and Streptomyces griseus, intracellular levels of ectoine increase in response to high growth temperature (Calderon et al., 2004; Malin and Lapidot, 1996). These findings suggest a role of ectoine as a protectant against the detrimental effects of high temperatures. In contrast, the ectoine biosynthesis in $V$. pantothenticus was triggered by low growth temperatures (Kuhlmann et al., 2008). The highest level of ectoine and the ectABC transcripts were detected $V$. pantothenticus cells subjected simultaneously to chilling $\left(15^{\circ} \mathrm{C}\right)$ and salt stress $(0.7 \mathrm{M} \mathrm{NaCl})$.

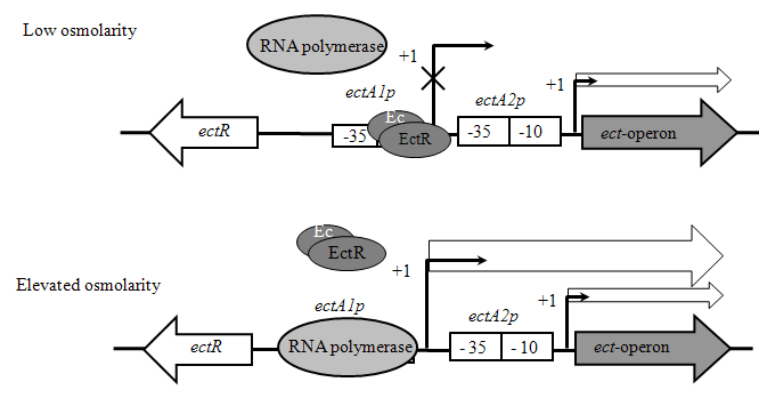

Fig. 3: Involvement of the EctR in transcriptional regulation of ectoine biosynthesis. At the low osmolarity, ectABC-ask genes of $\mathrm{Mm}$. alcaliphilum are constitutively transcribed from weak promoter ectA2p. EctR1 sterically inhibits binding of the RNA polymerase with 10 sequence of promoter ectA1p repressing transcription from osmoregulating promoter ectA1p. At the increasing of medium salinity, EctR-DNA complex is dissociated making promoter ectA1p to be accessible for RNA polymerase
Several additional regulatory elements of hydroxyectione biosynthesis can be recognized in $C$. salexigens and $S$. salexigens. In the latter, mRNA transcribed from the ectD promoter was 900 b.p. being larger than ectD sequence. An orf similar to MarRfamily of transcriptional regulators was revealed downstream of the ectD (Bursy et al., 2007). However, the putative regulatory protein is disrupted by two stop codons and no start codon could be found. In $C$. salexigens, the orf (gene ectR) was found upstream of ectD (Vargas et al., 2008; Garcia-Estepa et al., 2006). Deletion of the ectR gene resulted in decreasing of hydroxyectoine level at high salinity and high temperature, in comparison to the wild type cells. Thus, EctR (presumably belonging to the AraC family of transcriptional regulators) could be an activator of the ectD transcription.

Recent findings in transcriptional regulation of ectoine biosynthesis genes in methylotrophs: In $M$. alcaliphilum, transcription of the ectABC-ask operon is initiated from two $\sigma^{70}$-dependent promoters ectAp1 and ectAp2 (Fig. 3). The predicted -10 (TACTAT) and -35 (TGGACA) regions of ectAp1 showed rather high level of identity with the consensus sequence of the E. coli $\sigma^{70}$-recognized promoter (Reshetnikov et al., 2006; Mustakhimov et al., 2010). The putative -10 and -35 sequences of the ectAp2 promoter differ from the respective regions of the $E$. coli $\sigma^{70}$-promoter thus suggesting that expression from ectAp2 may be less effective, than from ectAp1.

Upstream of the ectA gene an orf (gene ectR1) encoding the MarR-like transcriptional regulator with 12$20 \%$ identity of translated amino acid sequences was found. Despite low identity, the protein has a structure analogous to the MarR-family regulators: it has the HelixTurn-Helix (HTH) DNA-binding motif flanking the "wing 1" region (Mustakhimov et al., 2010; Hong et al., 2005; Wilkinson and Grove, 2006). Regulatory function of EctR1 in ectoine biosynthesis was elucidated by characterization of the ectR1 knockout mutant. A promoter-reported system (ectP-gfp fusion) was constructed and introduced into $\mathrm{Mm}$. alcaliphilum wild type and mutant strains. The fluorescence signal in the strain lacking ectR1 was 2-3 folds higher compared to wild type cells. The DABA acetyltransferase activity was also 2-6 times higher in the mutant cells. Thus, EctR1 negatively controlled transcription of the ectgenes in Mm. alcaliphilum. Expression of the ectABCask operon in the ectR1 mutant was still activated by increasing of the medium salinity (from $1-6 \% \mathrm{NaCl}$ ) thus indicating that $\mathrm{Mm}$. alcaliphilum may possess several regulatory systems. 


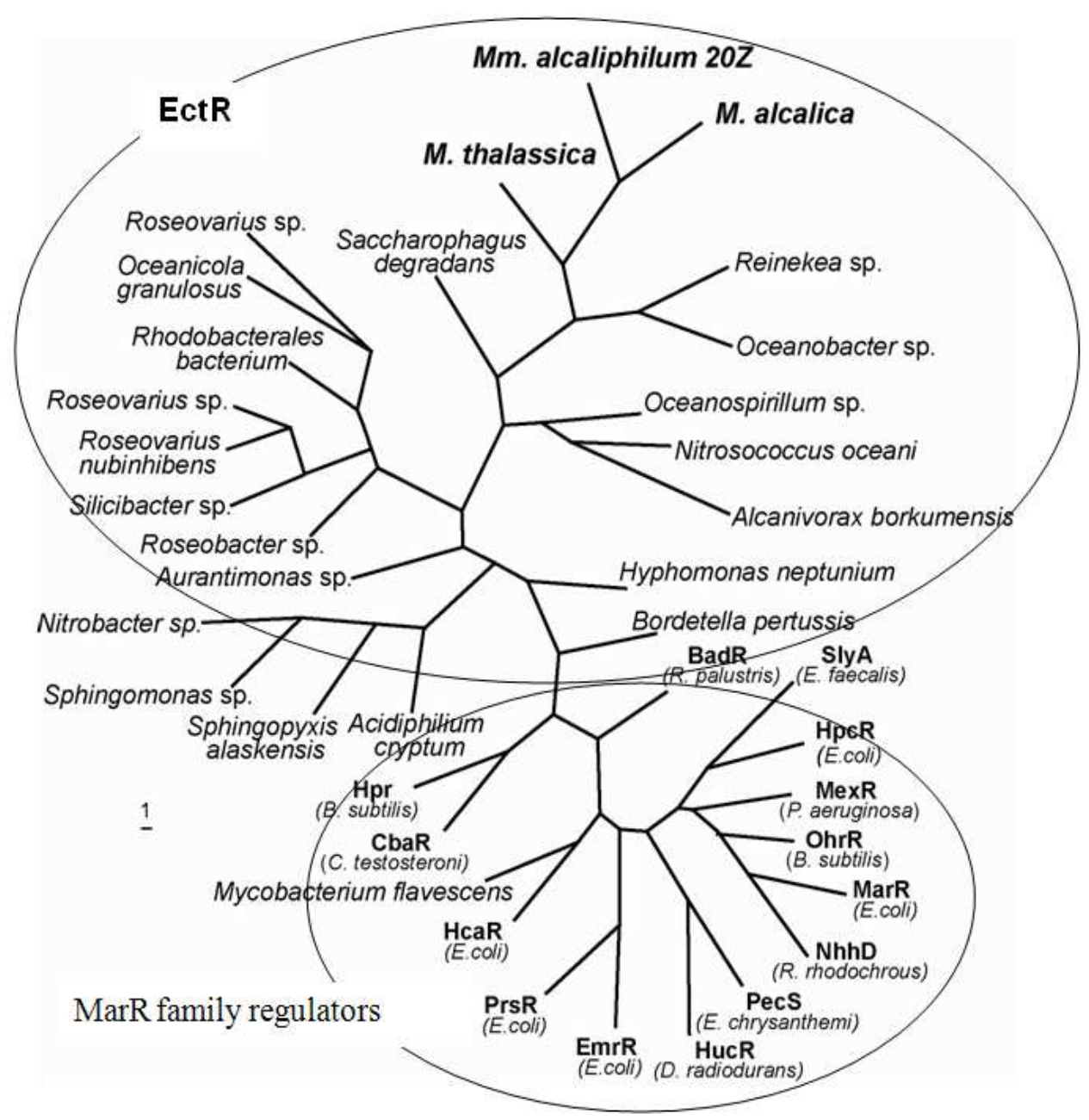

Fig. 4: Phylogenetic tree of putative transcriptional regulators EctR of halophilic bacteria and other regulators of the MarR-family

Transcription of the gene ectR1, in turn, was carried out from a single $\sigma^{70}$-like promoter (Fig. 3). The promoter region of ectR1, ectR1p, is located between ectAp1 and ectAp2, suggesting, that its transcription may be controlled by the EctR. The autoregulation was described for some other MarR proteins. The purified EctR1 specifically binds to the promoter region of ectABC-ask operon (Mustakhimov et al., 2010). The EctR1 binding site contains a pseudopalindromic sequence (TATTTAGT-GT-ACTATATA) composed of 8-bp half-sites separated by 2 bp suggesting dimeric association of the EctR1 with the DNA where each protein subunit binds with an inverted repeat (Mustakhimov et al., 2010). Indeed, gel-filtration studies showed that EctR is a dimer both in free solution (m.m. 44-45 kDa) and in DNA binding state (m.m. 50-55 kDa). Hence, it may be proposed that at the low osmolarity, the ectABC-ask genes of $\mathrm{Mm}$. alcaliphilum are constitutively transcribed from the weak promoter ectA2p (Fig. 3). EctR1 sterically inhibits the RNA polymerase binding with -10 sequence of the promoter ectA1p and thus represses transcription from the osmoregulating promoter ectA1p. At the elevated external salinity, EctR-DNA complex dissociates making the promoter ectA1p to be accessible for RNA polymerase (Fig. 3).

The EctR1 orthologs were identified in other halophilic bacteria. Our analysis of the DNA fragment containing the ectoine biosynthetic genes in the methanol-utilizing bacterium $M$. alcalica (Mustakhimov et al., 2009) showed the presence of an orf with high homology to the ectR1 gene from $\mathrm{Mm}$. alcaliphilum (73\% identity of translated amino acids). Moreover, a simple NCBI Blast search revealed several 
ectR1-like genes located immediately upstream of the ectoine gene cluster in 17 halophilic bacterial species (Fig. 4). Between them, the orfs of Oceanospirillum sp. (EAR60187), Nitrosococcus oceani (ABA57535), Saccharophagus degradans (ABD80450), Reinekea sp. (ZP_01114878) and Oceanobacter sp. (EAT11341) showed the highest identities of translated amino acid sequences with the EctR1 from Mm. alcaliphilum (35.5, 42.2 , 45.6, 51.7 and 55.1\%, respectively) which are higher than with other MarR-family transcriptional proteins $(<20 \%)$. We propose that putative EctR1 of these halophiles represents a separate subfamily in the MarR-family transcriptional proteins (Fig. 4). These results suggest that EctR-mediated regulatory system controlling ectoine biosynthesis at the transcriptional level may operate in diverse halophilic and halotolerant bacteria.

So far, it is not clear how changes in external osmolarity may regulate expression of the ectoine biosynthetic genes via the EctR1. It seems logical to propose that DNA binding ability depends on the external salinity. However, the activity of EctR1 could not be directly regulated by salt, since the expression of the ectABC-ask in Mm. alcaliphilum $20 \mathrm{Z}$ proceeds at low osmolarity. In any event, the tight relationship between salinity level and ectoine biosynthesis implies an involvement of complex multi tiered regulatory network, including inducers, sigma factors, transcriptional repressors and activators.

\section{CONCLUSION}

The necessity of unraveling the principles of organization and regulation of the genes and enzymes involved in ectoine biosynthesis by aerobic methylotrophs is conditioned by practical demands for a technology for production of the multifunctional valuable bioprotectants from cheap sources of carbon, such as methane and methanol. Large-scale production of ectoine has been achieved by the "Bitop" and "Merck" (Germany) using heterotrophic bacterium Halomonas elongata. This milking bioprocess is based on the use of the glucose, L-amino acids (glutamate) and highly saline medium $(12 \% \mathrm{NaCl}$ ) (Sauer and Galinski, 1998). Aerobic moderately halophilic and halotolerant methylotrophic bacteria when grown at 9\% $\mathrm{NaCl}$ are capable of accumulation ectoine up to $20 \%$ of DCW and therefore, are promising ectoine producers from renewable methane and/or methanol. The differences in ectoine accumulation may be caused by genetically defined regulatory mechanisms in the bacterial producers. Although the enzymology and genetics of the ectoine biosynthesis pathway in methylotrophs are similar to halophilic bacteria, they may have quite different regulatory patterns. For instance, the presence of the osmotically controlled aspartokinase in methylotrophs could make ectoine synthesis rather independent on other amino acids biosynthesis. Such co-ordination of aspartylphosphate synthesis, a common precursor of both ectoine and the amino acids, may provide an advantage during methylotrophic growth at high external osmolarity.

Osmoadaptation of aerobic methylotrophs, besides the osmoprotective compatible solutes biosynthesis (ectoine, glutamate and in some case sucrose), includes other structure-functional mechanisms such as changes in phospholipids fatty acids composition and in bioenergetic machinery. To date, it is not possible to describe whole regulatory cascade from sensing signals and receivers on a cell membrane to the real metabolic and structural rearrangements. Clarifying the nature of initial signals and sensors on a cell membrane and signal transduction to potential transcriptional regulators is a challenging task for future genomic transcriptomic and proteomic studies. The elucidation of cell responses to external perturbations, such as temperature, $\mathrm{pH}$ and others will shed more light on still enigmatic mechanisms of cross-adaptation of bacteria to fluctuating environmental conditions.

\section{ACKNOWLEDGMENT}

This study was supported by grants from the Russian Foundation for Basic Research (10-04-01224a; 09-04-92520-ИК_a), the Ministry of Education and Science RF (RNP2.1.1/605), Russian State Contract 02.740.11.0296, CRDF Rub1-2946-PU-09 and the U.S. National Science Foundation (MCB-0842686). The authors are gratefully acknowledged to the anonymous referees for useful comments and advices.

\section{REFERENCES}

Argandona, M, J.J. Nieto, F. Iglesias-Guerra, M.I. Calderon and R. Garcia-Estepa et al., 2010. Interplay between iron homeostasis and the osmotic stress response in the halophilic bacterium Chromohalobacter salexigens. Applied Environ. Microbiol., $\quad 76: \quad 3575-3589 . \quad$ DOI: 10.1128/AEM.03136-09

Bestvater, T. and E.A. Galinski, 2002. Investigation into a stress-inducible promoter region from Marinococcus halophilus using green fluorescent protein. Extremophiles, 6: 15-20. PMID: 11878557

Buenger, J. and H. Driller, 2004. Ectoin: An effective natural substance to prevent UVA-induced premature photoaging. Skin Pharmacol. Physiol., 17: 232-237. DOI: $10.1159 / 000080216$ 
Bursy, J., A.J. Pierik, N. Pica and E. Bremer, 2007. Osmotically induced synthesis of the compatible solute hydroxyectoine is mediated by an evolutionarily conserved ectoine hydroxylase. J. Biol. Chem., 282: 31147-31155. DOI: 10.1074/jbc.M704023200

Bursy, J., A.U. Kuhlmann, M. Pittelkow, H. Hartmann and M. Jebbar et al., 2008. Synthesis and uptake of the compatible solutes ectoine and 5hydroxyectoine by Streptomyces coelicolor A3(2) in response to salt and heat stresses. Applied Environ. Microbiol., 74: 7286-7296. DOI: 10.1128/AEM.00768-08

Calderon, M.I., C. Vargas, F. Rojo, F. Iglesias-Guerra and L.N. Csonka et al., 2004. Complex regulation of the synthesis of the compatible solute ectoine in the halophilic bacterium Chromohalobacter salexigens DSM 3043 ${ }^{\mathrm{T}}$. Microbiology, 150: 3051-3063. DOI: 10.1099/mic.0.27122-0

Canovas, D., C. Vargas, M.I. Calderon, A. Ventosa and J.J. Nieto, 1998. Characterization of the genes for the biosynthesis of the compatible solute ectoine in the moderately halophilic bacterium Halomonas elongata DSM 3043. Syst. Applied Microbiol., 21: 487-497. PMID: 9924816

Canovas, D., N. Borges, C. Vargas, A. Ventosa and J.J. Nieto et al., 1999. Role of $\mathrm{N} \gamma$ acetyldiaminobutyrate as an enzyme stabilizer and an intermediate in the biosynthesis of hydroxyectoine. Applied Environ. Microbiol., 65: 3774-3779. PMID: 10473374

Da Costa, M.S., H. Santos and E.A. Galinski, 1998. An overview of the role and diversity of compatible solutes in bacteria and archaea. Adv. Biochem. Eng. Biotechnol., 61: 117-153. PMID: 9670799

Doronina, N.V., Y.A. Trotsenko and T.P. Tourova, 2000. Methylarcula marina gen. nov., sp. nov. and Methylarcula terricola sp. nov.: Novel aerobic, moderately halophilic, facultatively methylotrophic bacteria from coastal saline environments. Int. J. Syst. Evol. Microbiol., 50: 1849-1859. PMID: 11034496

Doronina, N.V., T.D. Darmaeva and Y.A. Trotsenko, 2003a. Methylophaga alcalica sp. nov., a novel alkaliphilic and moderately halophilic, obligately methylotrophic bacterium from an East Mongolian saline soda lake. Int. J. Syst. Evol. Microbiol., 53: 223-229. DOI: 10.1099/ijs.0.02267-0

Doronina, N.V., T.D. Darmaeva and Y.A. Trotsenko, 2003b. Methylophaga natronica sp. nov., a new alkaliphilic and moderately halophilic, restrictedfacultatively methylotrophic bacterium from soda lake of the Southern Transbaikal region. Syst. Applied Microbiol., 26: 382-389. PMID: 14529181
Galinski, E.A., 1995. Osmoadaptation in bacteria. Adv. Microbiol. Physiol., 37: 273-328. PMID: 8540423

Galinski, E.A. and H.G. Truper, 1994. Microbial behavior in salt-stressed ecosystems. FEMS Microbiol. Rev., 15: 95-108. DOI: 10.1111/j.15746976.1994.tb00128.x

Galinski, E.A., H.P. Pfeiffer and H.G Truper, 1985. 1, 4,5,6, -Tetrahydro-2-methyl-4pyrimidinecarboxylic acid, a novel cyclic acid from halophilic phototrophic bacteria of the genus Ectothiorhodospira. Eur. J. Biochem., 149: 135-139. DOI: 10.1111/j.1432-1033.1985.tb08903.x

Garcia-Estepa, R., M. Argandona, M. Reina-Bueno, N. Capote and F. Iglesias-Guerra et al., 2006. The ectD gene, which is involved in the synthesis of the compatible solute hydroxyectoine, is essential for thermoprotection of the halophilic bacterium Chromohalobacter salexigens. J. Bacteriol., 188: 3774-3784. DOI: 10.1128/JB.00136-06

Gilboa, H., M. Kogut, S. Chalamish, R. Regev and Y. Avi-Dor et al., 1991. Use of ${ }^{23} \mathrm{Na}$ nuclear magnetic resonance spectroscopy to determine the true intracellular concentration of free sodium in a halophilic eubacterium. J. Bacteriol., 173: 7021-7023. PMID: 1938904

Goller, K., A. Ofer and E.A. Galinski, 1998. Construction and characterization of a NaClsensitive mutant of Halomonas elongata impaired in ectoine biosynthesis. FEMS Microbiol. Lett., 161: 293-300. PMID: 9570121

Graf, R., S. Anzali, J. Buenger, F. Pfluecker and H. Driller, 2008. The multifunctional role of ectoine as a natural cell protectant. Clin. Dermatol., 26: 326-333. DOI: 10.1016/j.clindermatol.2008.01.002

Grant, W.D., 2004. Life at low water activity. Phil. Trans. R. Soc. Lond. B., 359: 1249-1267. DOI: 10.1098/rstb.2004.1502

Hausinger, R.P., 2004. FeII/alpha-ketoglutaratedependent hydroxylases and related enzymes. Crit. Rev. Biochem. Mol. Biol., 39: 21-68. PMID: 15121720

Hong, M., M. Fuangthong, J.D. Helmann and R.G. Brennan, 2005. Structure of an OhrR-ohrA operator complex reveals the DNA binding mechanism of the MarR family. Mol. Cell, 20: 131-141. DOI: 10.1016/j.molcel.2005.09.013

Ikai, H. and S. Yamamoto, 1997. Identification and analysis of a gene encoding L-2,4-diaminobutyrate: 2-ketoglutarate 4-aminotransferase involved in 1,3diaminopropane production pathway in Acinetobacter baumannii. J. Bacteriol., 179: 5118-5125. PMID: 9260954 
Jebbar, M., R. Talibart, K. Gloux, T. Bernard and C. Blanco, 1992. Osmoprotection of Escherichia coli by ectoine: uptake and accumulation characteristics. J. Bacteriol., 174: 5027-5035. PMID: 1629159

Kalyuzhnaya, M.G., V.N. Khmelenina, B.T. Eshinimaev, N.E. Suzina and D. Nikitin et al., 2001. Taxonomic characterization of new alkaliphilic and alkalitolerant methanotrophs from soda lakes of the Southeastern Transbaikal region and description of Methylomicrobium buryatense sp. nov. Syst. Applied Microbiol., 24: 166-176. PMID: 11518319

Kalyuzhnaya, M.G., V. Khmelenina, B. Eshinimaev, D. Sorokin and H. Fuse et al., 2008. Classification of halo (alkali) philic and halo (alkali) tolerant methanotrophs provisionally assigned to the genera Methylomicrobium and Methylobacter and emended description of the genus Methylomicrobium. Int. J. Syst. Evol. Microbiol., 58: 591-596. DOI: 10.1099/ijs.0.65317-0

Kempf, B. and E. Bremer, 1998. Uptake and synthesis of compatible solutes as microbial stress responses to high-osmolality environments. Arch. Microbiol., 170: 319-330. DOI: 10.1007/s002030050649

Khmelenina, V.N., M.G. Kalyuzhnaya, V.G. Sakharovsky, N.E. Suzina and Y.A. Trotsenko et al., 1999. Osmoadaptation in halophilic and alkaliphilic methanotrophs. Arch. Microbiol., 172: 321-329. DOI: $10.1007 / \mathrm{s} 002030050786$

Kraegeloh, A. and H.J. Kunte, 2002. Novel insights into the role of potassium for osmoregulation in Halomonas elongata. Extremophiles, 6: 453-462. DOI: 10.1007/s00792-002-0277-4

Kuhlmann, A.U. and E. Bremer, 2002. Osmotically regulated synthesis of the compatible solute ectoine in Bacillus pasteurii and related Bacillus spp. Applied Environ. Microbiol., 68: 772-783. DOI: 10.1128/AEM.68.2.772-783.2002.

Kuhlmann, A.U., J. Bursy, S. Gimpel, T. Hoffmann and E. Bremer, 2008. Synthesis of the compatible solute ectoine in Virgibacillus pantothenticus is triggered by high salinity and low growth temperature. Applied Environ. Microbiol., 74: 4560-4563. DOI: 10.1128/AEM.00492-08

Lo, C.C., A.C. Bonner, G. Xie, M.D. Souza and A.R. Jensen, 2009. Cohesion group approach for evolutionary analysis of aspartokinase, an enzyme that feeds a branched network of many biochemical pathways. Microbiol. Mol. Biol. Rev., 73: 594-651. DOI: 10.1128/MMBR.00024-09
Louis, P. and E.A. Galinski, 1997. Characterization of genes for the biosynthesis of the compatible solute ectoine from Marinococcus halophilus and osmoregulated expression in Escherichia coli. Microbiology, 143: 1141-1149. DOI: 10.1099/00221287-143-4-1141

Malin, G. and A. Lapidot, 1996. Induction of synthesis tetrahydropyrimidine derivatives in Streptomyces strain and their effect on Escherichia coli in response to osmotic and heat stress. J. Bacteriol., 178: 385-395. PMID: 8550457

Marhuenda-Egea, F.C. and M.J. Bonete, 2002. Extreme halophilic enzymes in organic solvents. Curr. Opin. Biotechnol., 13: 385-389. DOI: 10.1016/S09581669(02)00338-5

McDonough, M.A., K.L. Kavanagh, D. Butler, T. Searls and U. Oppermann 2005. Structure of human phytanoyl-CoA2-hydroxylase identifies molecular mechanisms of Refsum disease. J. Biol. Chem., 280: 41101-41110. DOI: 10.1074/jbc.M507528200

Mevarech, M., F. Frolow and L.M. Gloss, 2000. Halophilic enzymes: Proteins with a grain of salt. Biophys. Chem., 86: 155-64. PMID: 11026680

Murrell, J.C., I.R. McDonald and B. Gilbert, 2000. Regulation of expression of methane monooxygenases by copper ions. Trends Microbiol., $\quad 8$ : 221-225. DOI: 10.1074/jbc.M507528200

Mustakhimov, I.I., O.N. Rozova, A.S. Reshetnikov, V.N. Khmelenina and J.C. Murrell et al., 2008. Characterization of the recombinant diaminobutyric acid acetyltransferase from Methylophaga thalassica and Methylophaga alcalica. FEMS Microbiol. Lett., 283: 91-96. DOI: 10.1111/j.1574-6968.2008.01156.x

Mustakhimov, I.I., A.S. Reshetnikov, V.N. Khmelenina and Y.A. Trotsenko, 2009. EctR-a novel transcriptional regulator of ectoine biosynthesis genes in the haloalcaliphilic methylotrophic bacterium Methylophaga alcalica. Dokl. Biochem. Biophys., 429: 305-308. PMID: 20101826

Mustakhimov, I.I., A.S. Reshetnikov, A.S Glukhov, V.N. Khmelenina and M.G. Kalyuzhnaya et al., 2010. Identification and characterization of EctR1, a new transcriptional regulator of the ectoine biosynthesis genes in the halotolerant methanotroph Methylomicrobium alcaliphilum 20Z. J. Bacteriol., 192: 410-417. DOI: 10.1128/JB.00553-09

Nagata, S., K. Adachi, K. Shirai and H. Sano, 1995. ${ }^{23} \mathrm{Na}$ NMR spectroscopy of free $\mathrm{Na}^{+}$in the halotolerant bacterium Brevibacterium sp. and Escherichia coli. Microbiolology, 141: 729-736. DOI: 10.1099/13500872-141-3-729 
Ono, H., K. Sawada, N. Khunajakr, T. Toa and M. Yamamoto et al., 1999. Characterization of biosynthetic enzymes for ectoine as a compatible solute in a moderately halophilic eubacterium, Halomonas elongata. J. Bacteriol., 181: 91-99. PMID 9864317

Onraedt, A., C. de muynck, B. Walcarius, W. Soetaert and E. Vandamme, 2004. Ectoine accumulation in Brevibacterium epidermis. Biotechnol. Lett., 26: 1481-1485. DOI: 10.1023/B:BILE.0000044448.86907.e4

Peters, R. and E.A. Galinski and H.G. Truper, 1990. The biosynthesis of ectoine. FEMS Microbiol. Lett., 71: 157-162. DOI: 10.1016/03781097(90)90049-V

Prabhu, J., F. Schauwecker, N. Grammel, U. Keller and M. Bernhard, 2004. Functional expression of the ectoine hydroxylase gene (thpD) from Streptomyces chrysomallus in Halomonas elongata. Applied Environ. Microbiol., 70: 3130-3132. DOI: 10.1128/AEM.70.5.3130-3132.2004

Rao, D.R., K. Hariharan and K.R. Vijayalakshmi, 1969. A study of the metabolism of L- $\alpha \gamma$-diaminobutyric acid in a Xanthomonas species. J. Biochem., 114: 107-115. PMID: 4390206

Reshetnikov, A.S., I.I. Mustakhimov, V.N. Khmelenina and Y.A Trotsenko, 2005. Cloning, purification and characterization of the diaminobutyrate acetyltransferase from the halotolerant methanotroph Methylomicrobium alcaliphilum 20Z. Biochemistry, 70: 878-883. PMID: 16212543

Reshetnikov, A.S., V.N. Khmelenina and Y.A. Trotsenko, 2006. Characterization of the ectoine biosynthesis genes of haloalkalotolerant obligate methanotroph "Methylomicrobium alcaliphilum 20Z". Arch. Microbiol., 184: 286-297. DOI: 10.1007/s00203005-0042-z

Reuter, K., M. Pittelkow, J. Bursy, A. Heine, T. Craan and E. Bremer, 2010. Synthesis of 5hydroxyectoine from ectoine: Crystal structure of the non-heme Iron(II) and 2-oxoglutarate-dependent dioxygenase EctD. PLoS One, 5: e10647-e10647. DOI: 10.1371/journal.pone.0010647

Roberts, M.F., 2004. Osmoadaptation and osmoregulation in archaea: Update. Front. Biosci., 9: 1999-2019. PMID: 15353266

Roberts, M.F., 2005. Organic compatible solutes of halotolerant and halophilic microorganisms. Saline Syst., 1: 1-5. DOI: 10.1186/1746-1448-1-5

Sauer, T. and E.A. Galinski, 1998. Bacterial milking a novel bioprocess for production of compatible solutes. Biotechnol. Bioeng., 57: 306-313. DOI: 10.1002/(SICI)10970290(19980205)57:3<306::AID-BIT7>3.0.CO;2-L
Saum, S.H. and V. Muller, 2007. Salinity-dependent switching of osmolyte strategies in a moderately halophilic bacterium: glutamate induces proline biosynthesis in Halobacillus halophilus. J. Bacteriol., 189: 6968-6975. DOI: 10.1128/JB.00775-07

Saum, S.H. and V. Muller, 2008a. Growth phasedependent switch in osmolyte strategy in a moderate halophile: Ectoine is a minor osmolyte but major stationary phase solute in Halobacillus halophilus. Environ. Microbiol., 10: 716-726. DOI: 10.1111/j.1462-2920.2007.01494.x

Saum, S.H and V. Muller, 2008b. Regulation of osmoadaptation in the moderate halophile Halobacillus halophilus: chloride, glutamate and switching osmolyte strategies. Saline Syst., 4: 1-4. DOI: $10.1186 / 1746-1448-4-4$

Severin, J., A. Wohlfarth and E.A. Galinski, 1992. The predominant role of recently discovered tetrahydropyrimidines for the osmoadaptation of halophilic eubacteria. J. Gen. Microbiol., 138: 1629-1638. DOI: 10.1099/00221287-138-81629

Toney, M.D., E. Hohenester, J.W. Keller and N. Jansonius, 1995. Structural and mechanistic analysis of two refined crystal structures of the pyridoxal phosphate-dependent enzyme dialkylglycine decarboxylase. J. Mol. Biol., 245: 151-179. DOI: 10.1006/jmbi.1994.0014

Vargas, C., M. Argandona, M. Reina-Bueno, J. RodriguezMoya and C. Fernandez-Aunion et al., 2008. Unraveling the adaptation responses to osmotic and temperature stress in Chromohalobacter salexigens, a bacterium with broad salinity tolerance. Saline Syst., 4: 1-14. DOI: 10.1186/1746-1448-4-14

Ventosa, A. and J.J. Nieto, 1995. Biotechological applications and potentialities of halophilic microorganisms. World J. Microbiol. Biotechnol., 11: 85-94. DOI: $10.1007 / \mathrm{BF} 00339138$

Wilkinson, S.P. and A. Grove, 2006. Ligand-responsive transcriptional regulation by members of the MarR family of winged helix proteins. Curr. Issues Mol. Biol., 8: 51-62. PMID: 16450885

Zhilina, T.N. and G.A. Zavarzin, 1990. Extremely halophilic, methylotrophic, anaerobic bacteria. FEMS Microbiol. Rev., 87: 315-322. DOI: 10.1111/j.1574-6968.1990.tb04930.x 